
\title{
28 Research Square \\ Tract Profiles of The Cerebellar Peduncles In Children Who Stutter
}

\section{Chelsea Johnson}

Michigan State University Department of Communicative Sciences and Disorders

Yanni Liu

University of Michigan

Noah Waller

University of Michigan

Soo-Eun Chang ( $\nabla$ sooeunc@med.umich.edu )

University of Michigan https://orcid.org/0000-0003-4448-9525

\section{Research Article}

Keywords: Stuttering, Cerebellum, diffusion MRI, fractional anisotropy, error monitoring

Posted Date: August 24th, 2021

DOl: https://doi.org/10.21203/rs.3.rs-682966/v1

License: (c) (i) This work is licensed under a Creative Commons Attribution 4.0 International License. Read Full License

Version of Record: A version of this preprint was published at Brain Structure and Function on February 27th, 2022. See the published version at https://doi.org/10.1007/s00429-022-02471-4. 


\section{Abstract}

Cerebellar-cortical loops comprise critical neural circuitry that supports self-initiated movements and motor adjustments in response to perceived errors, functions that are affected in stuttering. It is unknown whether structural aspects of cerebellar circuitry are affected in stuttering, in particular in children close to symptom onset. Here we examined white matter diffusivity characteristics of the three cerebellar peduncles (CP) based on diffusion MRI (dMRI) data collected from 41 children who stutter (CWS) and 42 controls in the 3-11 year range. We hypothesized that CWS would exhibit decreased fractional anisotropy (FA) in the right CPs given the contralateral connectivity of the cerebellar-cortical loops and past reports of structural differences in left cortical areas in stuttering speakers. Automatic Fiber Quantification (AFQ) was used to track and segment cerebellar white matter pathways and to extract diffusivity measures. We found significant group differences for FA in the right Inferior CP (ICP) only: controls showed significantly higher FA in the right ventral ICP compared to CWS, controlling for age, sex, and verbal IQ. Furthermore, FA of right ICP was negatively correlated with stuttering frequency in CWS. These results suggest an early developmental difference in the right ICP for CWS compared to age-matched peers, which may indicate an alteration in error processing, a function previously linked to the ICP. Lower FA here may impact error monitoring and sensory input processing to guide motor corrections. Further longitudinal investigations in children may provide additional insights into how CP development links to stuttering persistence and recovery.

\section{Introduction}

Stuttering is a complex neurodevelopmental disorder characterized by frequent disruptions in the flow of speech. Neuroimaging investigations in the last two decades have revealed significant differences in speakers who stutter relative to typical speakers in functional neuroanatomy affecting auditory-motor integration, temporal processing of speech movements, and aberrant inter-hemispheric interactions (for a review, see Chang et al., 2018). Apart from some convergent findings, there have been disparate reports of group differences that encompass many parts of the brain, reflecting the complex nature of stuttering and the probable involvement of multiple, network-level (versus region-specific) neural differences in stuttering. Examinations of the brain's "hub" areas that support the integration of functions across multiple neural structures have thus been an area of significant interest in the context of stuttering. One such area is the basal ganglia, which plays a major role in coordinating and initiating movement sequences and has been discussed in recent reviews relevant to the neural bases of stuttering (for a discussion, see Chang \& Guenther, Chang et al., 2018; Craig-McQuaide et al., 2014).

The cerebellum is another critical hub area of the brain (Akkal et al., 2007; Bostan et al., 2013; Bostan et al., 2010; Brodal, 1978; Glickstein et al., 1985; Hoover \& Strick, 1999; Hoshi et al., 2005; Kelly \& Strick, 2000, 2003; Leichnetz et al., 1984; Middleton \& Strick, 1994, 2001; Schmahmann \& Pandya, 1991, 1993, 1997; Snider \& Maiti, 1976; Strick et al., 2009; Tourville \& Guenther, 2011; Vilensky \& Van Hoesen, 1981; Zemanick et al., 1991). Arguably, the cerebellum has received much less exploration than other hub areas such as the basal ganglia, though a number of studies have reported cerebellar functional and structural 
differences in people who stutter (Beal et al., 2007; Brown et al., 2005; Budde et al., 2014; Chang et al., 2015; Chang et al., 2008, 2016a; Chang \& Zhu, 2013; Chow \& Chang, 2017; Connally et al., 2014; De Nil et al., 2001; Garnett et al., 2018; Kell et al., 2018; Lu et al., 2009, 2012; Chunming Lu, Chen, et al., 2010; Chunming Lu, Peng, et al., 2010a; Sitek et al., 2016; Song et al., 2007; Watkins et al., 2007, 2007; Yang et al., 2016). Several studies showed that people who stutter relative to controls exhibit greater activity in the right cerebellum during speech tasks (De Nil et al., 2001, 2003; Ingham et al., 2012; Lu, Peng, et al., 2010b; Watkins et al., 2007). The cerebellar right hemisphere areas primarily inter-connect with left cerebral hemisphere regions, supporting functions including orofacial movements and speech motor control. In addition to motor functions, recent studies have provided strong evidence that the cerebellum is substantially involved in supporting higher-order cognitive processes. For example, working memory, semantic judgment, spatial awareness, procedural learning, decision making, and emotion processing all engage the cerebellum (for a review, see Stoodley \& Schmahmann, 2010). Given updated understanding of cerebellar function and cerebellar networks, further research into cerebellar functional neuroanatomy is expected to lead to a better understanding of the neural bases underlying the multifactorial nature of stuttering (for a discussion, see Smith \& Weber, 2017).

Major white matter tracts that interconnect the cerebellum and the cerebrum and brainstem are bundled into cerebellar peduncles. There are 3 pairs of cerebellar peduncles: The superior cerebellar peduncles (SCP), which contain almost all of the efferent projections from the cerebellum via the thalamus, such as those projecting to cortical motor areas; the middle cerebellar peduncles (MCP), which are the primary afferent pathway carrying input from the cortex to the cerebellum via the pons; and the inferior cerebellar peduncles (ICP), which carry both afferent and efferent tracts. Afferent pathways that travel to the ICP originate in vestibular areas, the spinal cord, and the brainstem. Efferent channels from this peduncle also travel to vestibular regions and brainstem areas that mediate vital life functions, such as circadian rhythm and consciousness, as well as reflexes (e.g., Mangold \& Das, 2020). The ICP have also been associated with sensorimotor adaptation (Jossinger et al., 2020) and error detections during motor commands (for a review, see Shadmehr, 2017). Relevant to speech-motor control theories, afferent information from the olivo-cerebellar fibers that enter the cerebellum through the ICP provide the cerebellum with sensory input from the periphery (Streng et al., 2018). This peripheral information could help guide the proper updating of motor commands through efferent copy mechanisms that are supported by the cerebellum (e.g., De Zeeuw et al., 1998; Miall et al., 1993; Rhodes \& Bullock, 2002; Wolpert et al., 1998)

Adults and children with persistent stuttering differ in microstructural characteristics of the cerebellar peduncles compared to controls and recovered children who stutter (Chow \& Chang, 2017; Connally et al., 2014; Garnett et al., 2018; Watkins et al., 2007). However, there is some disagreement on which peduncles may be affected in developmental stuttering. While some studies have suggested that all six peduncles have lower FA in adults who stutter (Connally et al., 2014), other studies have only found the right middle peduncle to differ significantly in adults who stutter relative to controls (Watkins et al., 2007). Connally et al. (2014) also showed a significant age effect in the FA of the ICP: FA in ICP increased with age (14-42 years) in speakers who stutter only, whereas there were no such age correlations in the controls. Another 
study found that while FA of the left ICP was associated with speech rate in adults who stutter, adults who stutter did not differ from controls in overall structural measures of any of the cerebellar peduncles (Jossinger et al., 2021). Both the inferior and middle cerebellar peduncles carry afferent signals from speech motor control substrates in the brainstem and the cerebral cortex, respectively. Given that there are some controversies surrounding whether speech motor learning differences exist in adults but not in children who stutter (Daliri et al., 2017; Kim et al., 2020), it is of interest to examine whether subtle differences reported in cerebellar peduncle structure in adults who stutter are also present in children who stutter. Examining children who stutter, who are less likely than in adults to have acquired adaptive neuroplastic changes associated with reactions and compensations to one's own stuttering, is critical to be able to attribute any structural changes to be associated with stuttering itself, or to adaptive changes.

In the above-mentioned studies, diffusion MRI (dMRI) was used to quantify water diffusion, reflecting brain tissue organization on a microstructural scale. The white matter structures inhabited by myelinated axons induce an anisotropic diffusion of water molecules, compared to gray matter structures or in the cerebrospinal fluid, where water diffusion would be much more isotropic (i.e., mean diffusion probability occurring equally in all directions rather than in a preferential direction). Fractional anisotropy (FA) is a summary measure derived from dMRI that provides a way to quantify how much of the water diffusion occurs in a principal direction compared to orthogonal directions. FA is influenced by various factors such as myelination, axonal diameter, presence of crossing fibers, and is commonly used in clinical studies and discussed as a measure reflecting white matter "integrity" or "coherence". While FA can be examined using a voxel-based analysis (e.g., TBSS; Smith et al., 2006) to compare between groups, there are some limitations in terms of ensuring that TBSS-based tracts correspond to tracts in individual brains (Tsang et al., 2010; Yeatman et al., 2012). Compared to voxel-wise analyses of FA, tractography involves probabilistic or deterministic algorithms to track the continuous trajectory of white matter tracts and is considered a more accurate method to identify white matter tracts in the human brain (Yeatman et al., 2012). However, most common methods for tractography investigations have relied on manual ROI definitions that were effortful and time-consuming. Furthermore, diffusion properties of tracts were often averaged along the length of the whole tract.

A new tractography method, Automated Fiber Quantification (AFQ), improves upon these limitations and provides a way to automatically identify major white matter tracts (Yeatman et al., 2012). AFQ also allows examining diffusion measurements at different locations on the tracts rather than just the mean across the entire tract. While this method has been applied to study adults who stutter (Jossinger et al., 2021; Kronfeld-Duenias et al., 2016a, 2016b, 2018), it has not yet been applied to study white matter tracts in children who stutter who are relatively close to stuttering onset. Using this method, we examined five major cerebellar white matter tracts in children who stutter and their age-matched peers to compare diffusion properties at anatomically equivalent locations of each tract. These tracts included the left and right superior cerebellar peduncles, the middle cerebellar peduncle, and the left and right ICPs.

We hypothesized that children who stutter would exhibit decreased FA in the right cerebellar peduncles, given the contralateral connectivity of the cerebellar cortical loops and previously reported convergent 
findings of differences focused on the left cerebral areas in people who stutter. Given the reported role of the ICP in error detection, and its role in carrying afferent fibers to the cerebellum with peripheral sensory information that may help support efference copy mechanisms during speech (e.g., Shadmehr, 2017; Streng et al., 2018), we hypothesized that the right inferior peduncle would be associated with persistent stuttering and stuttering severity.

\section{Methods}

\subsection{Participants}

A total of 83 children ( 41 stuttering, 26 boys; 42 controls, 21 boys) between 3 and 11 years of age participated. All were monolingual native North American English speakers without concomitant developmental disorders (e.g., dyslexia, attention-deficit/hyperactivity disorder, learning delay, psychiatric conditions). All children underwent careful screening to ensure typical speech and language developmental history except for the presence of stuttering in the experimental groups. The children who stutter and controls were matched in age, handedness (Oldfield, 1971), and socioeconomic status (Hollingshead, 1975). While most participants were strongly right-handed, 6 children were left-handed (2 persistent, 1 recovered, 3 control) and 7 ambidextrous ( 4 persistent, 1 recovered, 2 control). All participants were tested on a battery of standardized speech, language, and cognitive tests, audiometric hearing screening, oral-motor screening, and cognitive evaluations. The tests included the Peabody Picture Vocabulary Test (PPVT-3; Dunn, Dunn, \& Lenhard, 2007), Expressive Vocabulary Test (EVT-2; Williams \& Williams, 2007), Goldman-Fristoe Test of Articulation (GFTA-2; Goldman, 2000), Wechsler Preschool and Primary Scale of Intelligence (WPPSI-III; for children 2: 6-7:3; Wechsler, 2002), and Wechsler Abbreviated Scale of Intelligence (WASI; for children aged 7 and up; Wechsler, 1999). Children were excluded if scores fell below two standard deviations (SD) of the mean on any standardized assessments. The average test scores for each group are listed in Table 1.

Stuttering severity was assessed by collecting samples of spontaneous speech elicited through storytelling and conversational tasks with a parent and a certified speech-language pathologist. These samples were video recorded for further offline analyses. We calculated percent stuttered utterances per number of syllables based on narrative samples containing a conversation with the clinician and a monologue elicited with storytelling with a pictures-only book ('Frog, where are you?'; Mayer, 1969). In addition, the Stuttering Severity Instrument (SSI; Riley, 2009) was used to examine the frequency and duration of disfluencies occurring in the speech sample, as well as any physical concomitants associated with stuttering; all of these measures were incorporated into a composite stuttering severity rating. To determine the measurement reliability of the SSI scores, an intraclass correlation coefficient was calculated based on the ratings from two independent judges on children's speech samples.

While all children who stutter were diagnosed with stuttering at the initial study visit, they were categorized as recovered or persistent based on measurements acquired in subsequent early visits that occurred up to 4 times for each child. A child was categorized as persistent with an SSI- 4 score $>10$ at 
two consecutive follow-up visits, and the onset of stuttering had been at least 36 months prior to his most recent visit. A child was considered recovered with an SSI-4 score $\leq 10$ (corresponding to "very mild") at two consecutive follow-up visits. Such determination also required the consideration of percent occurrence of stuttering-like disfluencies (\%SLD) in the speech sample ( $\geq 3$ for persistent) as well as clinician and parental reports. Similar criteria were used to determine recovery or persistency in previous studies (Yairi \& Ambrose, 1999). Using these criteria, we identified 13 recovered children (7 boys), hereafter "recovered" and 28 persistent children (18 boys), hereafter "persistent". For controls, the inclusion criteria included never having been diagnosed with stuttering, no family history of stuttering, lack of parental concern for their child's speech fluency, with \%SLD $<3 \%$.

Table 1 Demographic information and behavioral test scores of children who stutter and control participants included in this study 
Persistent

Controls

$n=42(21$

boys)
Recovered

$n=13(8$

boys)

\begin{tabular}{|c|c|c|c|c|c|c|}
\hline & Mean (SD) & Range & Mean (SD) & Range & Mean (SD) & Range \\
\hline Age & $6.52(2.03)$ & $\begin{array}{l}3.25- \\
10.75\end{array}$ & $6.52(1.95)$ & $\begin{array}{l}3.08- \\
10.33\end{array}$ & $\begin{array}{l}5.77 \\
(2.31)\end{array}$ & $\begin{array}{l}3.66- \\
11.00\end{array}$ \\
\hline $\begin{array}{l}\text { SES (Maternal } \\
\text { Education) }\end{array}$ & $6.36(0.61)$ & $5-7$ & $6.21(0.83)$ & $4-7$ & $\begin{array}{l}6.30 \\
(0.63)\end{array}$ & $5-7$ \\
\hline Full-Scale IQ ${ }^{a}$ & $\begin{array}{l}114.68 \\
(14.33)\end{array}$ & $84-144$ & 106.78(14.30) & $81-138$ & $\begin{array}{l}104.00 \\
(15.47)\end{array}$ & $88-130$ \\
\hline Performance IQ & $\begin{array}{l}111.58 \\
(15.92)\end{array}$ & $77-145$ & $\begin{array}{l}107.85 \\
(13.24)\end{array}$ & $79-135$ & $\begin{array}{l}101.53 \\
(14.91)\end{array}$ & 86- 134 \\
\hline Verbal IQ ${ }^{a}$ & $\begin{array}{l}117.04 \\
(14.93)\end{array}$ & $87-153$ & $\begin{array}{l}105.32 \\
(13.83)\end{array}$ & $\begin{array}{l}77- \\
137\end{array}$ & $\begin{array}{l}106.00 \\
(15.54)\end{array}$ & $88-136$ \\
\hline PPVT $^{\mathrm{c}}$ & $\begin{array}{l}118.26 \\
(13.61)\end{array}$ & $95-151$ & $\begin{array}{l}109.79 \\
(12.33)\end{array}$ & $87-147$ & $\begin{array}{l}110.69 \\
(15.44)\end{array}$ & $86-134$ \\
\hline $\mathrm{EVT}^{\mathrm{c}}$ & $\begin{array}{l}115.65 \\
(14.33)\end{array}$ & $90-149$ & $\begin{array}{l}106.57 \\
(11.66)\end{array}$ & $89-134$ & $\begin{array}{l}106.84 \\
13.94)\end{array}$ & $87-137$ \\
\hline GFTA & $\begin{array}{l}105.31 \\
(7.95)\end{array}$ & $81-123$ & $101.86(8.58)$ & $77-118$ & $\begin{array}{l}107.38 \\
(8.32)\end{array}$ & $96-121$ \\
\hline$\% S L D^{b}$ & $1.08(0.87)$ & $0.0-3.23$ & $6.88(6.55)$ & $\begin{array}{l}1.10- \\
30.20\end{array}$ & $\begin{array}{l}4.62 \\
(3.33)\end{array}$ & $\begin{array}{l}0.20- \\
12.0\end{array}$ \\
\hline$\% O D$ & $4.93(2.67)$ & $\begin{array}{l}0.0- \\
13.73\end{array}$ & $5.61(2.91)$ & $\begin{array}{l}1.00- \\
12.70\end{array}$ & $\begin{array}{l}4.60 \\
(1.96)\end{array}$ & $\begin{array}{l}2.20- \\
9.0\end{array}$ \\
\hline SSI-4 at initial visit & $\mathrm{N} / \mathrm{A}$ & $\mathrm{N} / \mathrm{A}$ & $21.18(7.96)$ & $12-48$ & $\begin{array}{l}16.00 \\
(6.00)\end{array}$ & $6-28$ \\
\hline
\end{tabular}

${ }^{a}$ Controls exhibited significantly higher scores than the persistent and recovered CWS groups.

${ }^{b}$ Both persistent and recovered groups exhibited significantly higher scores than in controls.

${ }^{c}$ Controls exhibited significantly higher scores than the persistent CWS group.

SD, standard deviation; SES, socioeconomic status; IQ, intelligence quotient; PPVT, Peabody Picture Vocabulary Test; EVT, Expressive Vocabulary Test; GFTA, Goldman-Fristoe Test of Articulation; SSI-4, Stuttering Severity Instrument Edition 4; \%SLD, stuttering-like disfluencies (e.g., sound-syllable repetitions, word repetitions, sound prolongations) occurring per 100 words during conversational speech; \%OD, other 
disfluencies (e.g., interjections, phrase repetitions) occurring per 100 words during conversational speech.

In addition to the speech-language and cognitive tests, all children went through mock scanner training during a separate visit to familiarize them to the scanner environment and procedures and to practice keeping still while lying inside the bore for stretches of time. Recordings of MRI scanner noise were played during this session so that children were aware that they would hear loud sounds during scanning. This session was repeated in some children as needed. All children were paid a nominal remuneration and were given small prizes (e.g., stickers) for their participation. All procedures used in this study were approved by the Michigan State University Institutional Review Board.

\subsection{MRI acquisition}

MRI scans for this study were acquired on a GE 3T SignaVR HDx MR scanner (GE Healthcare) with an 8channel head coil. During each scanning session, 180 T1-weighted 1-mm3 isotropic volumetric inversion recovery fast spoiled gradient-recalled images, with CSF suppressed, were collected to cover the entire brain with the following parameters: time of echo $3.8 \mathrm{~ms}$, time of repetition of acquisition $8.6 \mathrm{~ms}$, time of inversion $831 \mathrm{~ms}$, repetition time of inversion 2,332 ms, flip angle 8 degrees, and receiver bandwidth $+/-20.8 \mathrm{kHz}$. After collecting T1 data, high-order shimming procedures were performed to improve magnetic field homogeneity. The dMRI data were obtained with a dual spin-echo echo-planar imaging sequence for 12 minutes and 6 seconds with the following parameters: 48 contiguous 2.4-mm axial slices in an interleaved order, field of view $22 \times 22 \mathrm{~cm}$, matrix size $128 \times 128$, number of excitations =2, echo time $77.5 \mathrm{~ms}$, repetition time $13.7 \mathrm{~s}, 25$ diffusion-weighted volumes (one per gradient direction) with $b=1,000 \mathrm{~s} / \mathrm{mm} 2$, one volume with $b=0$ and parallel imaging acceleration factor $=2$. A member of the research staff sat inside the scanner room next to the child being scanned for the duration of the scans to monitor participant comfort and to ensure the child was able to cooperate with scanning protocols. Children watched a movie to help them stay still during the acquisition of volumetric T1-weighted scans and dMRI scans.

\subsection{Diffusion MRI data preprocessing}

Diffusion MRI data were preprocessed individually using the open-source software mrDiffusion (https://github.com/vistalab/vistasoft/tree/master/mrDiffusion) implemented in MATLAB R2017b. Eddy current distortions and subject motion in the diffusion-weighted images were removed by a 14-parameter constrained non-linear co-registration algorithm based on the expected pattern of eddy current distortions (Rohde et al., 2004). Each diffusion-weighted image was registered to the non-diffusion (b0) image; the b0 image was registered automatically to the T1 image, which had been aligned to the canonical MNI template. The combined transformation, incorporating both eddy-current correction and anatomical alignment, was applied to the raw diffusion data, and the transformed images were resampled at $2 \times 2 \times 2 \mathrm{~mm}$ isotropic voxels. Diffusion tensors were then fit using a robust least-squares algorithm. Eigenvectors and eigenvalues of the tensor were extracted. FA was calculated as the normalized standard deviation of the eigenvalues of the diffusion tensor, and MD was calculated as the 
average of all three eigenvalues (Pierpaoli \& Basser, 1996). Head motion was quantified in each participant by calculating the degree of motion correction in each volume relative to prior volume (Bruckert et al., 2019). Average displacement across participants $0.046+/-0.030$, and there was no group difference in the displacement $(p>0.05)$.

\subsection{Tract identification and segmentation}

Five cerebellar peduncles (CPS) (bilateral ICP, bilateral SCP, and MCP) were identified and quantified using the open-source software Automated Fiber Quantification (AFQ) package (Yeatman et al., 2012). AFQ consists of three main processing steps: (1) whole-brain fiber tractography, (2) automatic tract segmentation based on template region of interest (ROIs) warped to native space, and (3) automatic tract quantification and cleaning. A whole-brain fiber group was tracked using a deterministic streamlines tracking tractography (STT) algorithm (Mori et al. 1999). Based on previous dMRI studies in children (Bruckert et al., 2019), the tracking algorithm was seeded with a white matter mask defined as all the voxels with FA value greater than 0.15 . Tracking proceeded in both directions along the principal diffusion axes and stopped when FA estimated at the current position dropped below 0.10 or when the angle between the last path segment and next step direction was greater than $30^{\circ}$. Fiber tract segmentation was based on waypoint ROIs, which were first defined on the JHU MNI T1 template and then back-transformed to each participants' native space (Bruckert et al., 2019; Jossinger et al., 2021). The core of the tract was calculated by defining 30 sample points along the tract and computing the robust mean position of the corresponding sample points. After tract segmentation, an automated cleaning algorithm was used to remove fiber longer than 1 standard deviation from the mean fiber length and spatially deviated more than 4 standard deviations from the core of the tract.

A small number of children who stutter were excluded from each analysis because a tract could not be segmented or did not conform to anatomical norms. One child who stutters was excluded from the MCP, 1 from the right SCP, and 2 from the left SCP analyses. Diffusion properties (FA, MD) were calculated at 30 equidistant nodes along the central portion of each fiber tract bounded by the same two waypoint ROls used for tract segmentation (Bruckert et al., 2019; Jossinger et al., 2021; Yeatman et al., 2012). Figure $\mathrm{S} 1$ shows the tracts of interest identified in one representative participant and visualization of FA profiles in each group and each CP.

\subsection{Statistical analysis}

Analyses were conducted on the core of each CP tract between the two waypoint-ROIs used for tractography.

Group differences. Mean tract-diffusion indices (FA and MD) were calculated by averaging values of all 30 nodes for each participant and in each peduncle (hereafter referred to as "tract-FA and tract-MD"). Independent t-tests were used to compare tract-FA and tract-MD between groups (CWS, controls) in the MCP, bilateral ICP, and SCP. In addition, we evaluated group differences of the local diffusivity measures along the 30 nodes in each tract (hereafter, node_FA, node_MD), which may provide better sensitivity 
compared to the mean FA and MD for the whole tract. These along-tract group comparison statistics were corrected using a non-parametric permutation test $(p<0.05)$ and controlling the family-wise error $(F W E)$ corrected alpha at 0.05 (Nichols \& Holmes, 2002).

Linear regression and correlations. To account for the additional factors that could contribute to the group differences of diffusion properties in the CPs, a linear regression model was built to test group differences when age, sex, and IQ were included as covariates. Meanwhile, Pearson correlation coefficients were used to examine age correlations with diffusion properties of CPs and to test whether diffusion properties of CPs showing significant group differences were associated with stuttering severity.

\section{Results}

\subsection{Reduced white matter integrity in the right inferior cerebellar peduncle (RICP) in children who stutter.} Mean tract_FA and tract_MD for each cerebellar peduncle tract are shown in Table 2 and Fig. 1. CWS showed reduced FA in right ICP $(\mathrm{p}<0.013)$ and showed a trend-level significance of greater MD in MCP compared to controls $(p=0.051$; Table 2$)$. There were no group differences in diffusivity values of any other tracts. We also tested tract profiles in local diffusivity measures (node_FA, node_MD) and found FA group differences between CWS and controls in the ventral nodes of the right ICP ( $p<0.05$, corrected for multiple comparisons, see Fig. 2 node 3-12). Post-hoc t-test results show that there were group differences between persistent CWS and controls for both tract_FA $(p=0.003)$ and node_FA ( $p<0.001)$; but no group differences were found between recovered CWS and controls, or persistent CWS and recovered CWS.

Table 2 Fractional anisotropy (FA) and mean diffusivity (MD) profiles in each cerebellar tract examined for controls and children who stutter (CWS). Significant group differences were present only for the right ICP. 
Controls CWS Group Difference Age Correlation

\begin{tabular}{lllllllll}
\hline & mean & $\pm S D$ & mean & SD & $t$ & $p$ & $r$ & $p$ \\
\hline rightICP_FA & 0.475 & 0.042 & 0.451 & 0.046 & 2.544 & $\mathbf{0 . 0 1 3}$ & 0.283 & $\mathbf{0 . 0 0 9 *}$ \\
\hline rightICP_MD & 0.883 & 0.182 & 0.869 & 0.074 & 0.445 & 0.657 & -0.116 & 0.298 \\
\hline leftICP_FA & 0.460 & 0.049 & 0.450 & 0.049 & 0.925 & 0.358 & 0.320 & $\mathbf{0 . 0 0 3 *}$ \\
\hline leftICP_MD & 0.873 & 0.107 & 0.859 & 0.060 & 0.706 & 0.482 & -0.152 & 0.172 \\
\hline rightSCP_FA & 0.378 & 0.054 & 0.383 & 0.045 & -0.476 & 0.636 & 0.330 & $\mathbf{0 . 0 0 3 *}$ \\
\hline rightSCP_MD & 1.245 & 0.208 & 1.242 & 0.193 & 0.072 & 0.943 & -0.267 & $\mathbf{0 . 0 1 6 *}$ \\
\hline leftSCP_FA & 0.368 & 0.048 & 0.372 & 0.052 & -0.317 & 0.752 & 0.255 & $\mathbf{0 . 0 2 1 *}$ \\
\hline leftSCP_MD & 1.186 & 0.228 & 1.176 & 0.169 & 0.218 & 0.828 & -0.075 & 0.508 \\
\hline MCP_FA & 0.441 & 0.057 & 0.435 & 0.060 & 0.518 & 0.606 & 0.160 & 0.150 \\
\hline MCP_MD & 1.022 & 0.123 & 1.088 & 0.178 & -1.985 & $0.051 \#$ & -0.165 & 0.140
\end{tabular}

CWS = children who stutter; ICP = Inferior Cerebellar Peduncle, $\mathrm{MCP}=$ Middle Cerebellar Peduncle, $\mathrm{SCP}=$ Superior Cerebellar Peduncle; $S D=$ standard deviation; $L=$ left; $R=$ right. ${ }^{*} p<0.05 ; \# p<0.10$

\subsection{White matter integrity of the RICP scales with age and stuttering severity.}

Table 3 shows results from the linear regression models where group, age, sex, and IQ were included as covariates, and mean FA of the whole right ICP tract (Tract_FA), and mean FA of nodes 3 to 12 in the right ICP showing significant group difference (Node_FA) were dependent variables. Group differences were still significant for both tract_FA and node_FA in the right ICP when controlling for age, sex, and IQ. Meanwhile, in both models, age was positively associated with FA: Age was correlated with mean Node_FA for nodes 3-12 and mean FA in the whole tract of the right ICP (Fig. 3). Furthermore, we found that mean Tract_FA of the right ICP in children who stutter was significantly correlated ( $r=-0.315$., $p=0.045)$ with stuttering frequency (\%SLD) (Fig. 4). The correlation was still significant when controlling for age $(r=-0.314, p=0.049)$. No significant correlation was found between Node_FA of the right ICP and stuttering frequency $(\% S L D)(r=-0.203, p=0.203)$.

In addition to the right ICP FA, significant correlation of age and mean tract FA were observed regardless of group in the left ICP, bilateral SCPs, and MD of the right SCP (Table 2). 
Table 3. Regression analysis for the prediction of tract_FA and node_FA of the right ICP. In addition to significant group differences found in both node_and tract_FA for the right ICP, there was a significant effect of age for this same tract regardless of group.

\begin{tabular}{|c|c|c|c|c|c|c|}
\hline & \multicolumn{3}{|c|}{ Node_FA } & \multicolumn{3}{|c|}{ Tract_FA } \\
\hline & beta & $\mathrm{t}$ & $p$ & beta & $\mathrm{t}$ & $\mathrm{p}$ \\
\hline Group & -0.326 & -3.004 & $0.004^{*}$ & -0.240 & -2.127 & 0.037 * \\
\hline Sex & -0.021 & -0.206 & 0.837 & -0.089 & -0.830 & 0.409 \\
\hline Age & 0.303 & 2.942 & $0.004^{*}$ & 0.258 & 2.413 & $0.018 *$ \\
\hline IQ & 0.026 & 0.237 & 0.813 & 0.013 & 0.113 & 0.911 \\
\hline
\end{tabular}

\section{Discussion}

With its vast connections with cerebral structures associated with motor and cognitive functions and its well-known critical role in supporting motor control, particularly in finely timed self-initiated movements such as speech, the cerebellum is an important yet understudied area of interest in the context of stuttering. To date, only a handful of studies have focused on examining cerebellar function and anatomy, all in adults and adolescents who stutter, with few studies reported to date in children who stutter. The current study examined white matter microstructural changes associated with childhood stuttering based on diffusion-weighted MRI data. The Automated Fiber Quantification (AFQ) tractography method was used to overcome limitations of previous dMRI methods, to examine FA and MD derived from the core of major white matter tracts, both at the mean tract level as well as at specific nodes along each tract. The main results were that children who stutter have reduced FA in the right ICP of the cerebellum relative to controls. Across the right ICP, children who stutter had significantly lower FA, particularly in the ventral nodes of this cerebellar peduncle. Right ICP FA was also negatively correlated with stuttering frequency in children who stutter. Overall, across both groups, FA in the right ICP was positively correlated with age. There were no significant group differences based on MD.

These results, derived from the novel application of AFQ methods to study children who stutter, provide additional insight into reports of cerebellar peduncle microstructural differences described in previous studies of stuttering. Though one study examining persistent children who stutter compared to controls found that there was a significant age $x$ group interaction of FA in the right ICP such that the growth rate was less in persistent children who stutter than controls (Chow \& Chang, 2017), other ICP findings based on examining adults who stutter have been inconsistent. In one study, lower FA was found in all three pairs of cerebellar peduncles for speakers who stutter (age range: $14-42 ; \mathrm{N}=29$ ) relative to controls (age range: $14-45 ; N=37$ ), with the greatest group difference occurring in the left ICP (Connally et al., 2014). Another study reported lower FA in the right middle cerebellar peduncle (MCP) in speakers who stutter 
(age range: $14-27$ years, $N=17$; control group age range: $14-27$ years, $N=13$ ) (Watkins et al., 2007), and in another, no differences were found between the stuttering and control groups, but the left ICP FA was negatively correlated with speech rate in adults who stutter (age range: 19-52 years, $\mathrm{N}=23$; control group age range: $19-53$ years, $N=19$ ) (Jossinger et al., 2021). The discrepancies reported by the studies mentioned above may be explained by differences in the age range of participants examined, varying statistical power to detect group differences, as well as differing scanning protocols and dMRI analysis methods applied in the analyses (for a discussion of the latter point, see Jossinger, 2021).

Concerning age, Jossinger et al. (2021) point out that group differences reported by Connally et al. (2014) for FA of the ICP decreased dramatically with age. This result may suggest that white matter development for people who stutter (PWS) in ICP may be delayed but eventually catch up to levels seen in controls during adulthood. Jossinger et al. also did not find significant group differences in the ICP in their participants who had a higher mean age than in the other two studies. The current study is the first to examine $\mathrm{CP}$ white matter microstructure in children ranging from 3-11 years of age, with the largest sample size reported of the studies published to date. We found significant group differences in FA of the right ICP only and age-related increases in this same tract for both groups, which offers some clarifications on the early development of the CPs that differentiate PWS from age-matched peers. Like Jossinger et al. (2021) that used identical methods as the current study employing AFQ, we did not find significant group differences in any of the diffusivity measures in the MCP (only trend level increase in MD found for CWS relative to controls), bilateral SCP, or left ICP. Unlike Jossinger et al. (2021), we found group differences in the right ICP where they did not find a significant group difference; they only reported a relationship between FA in the left ICP with speech rate in PWS. Findings from the two studies collectively point to the ICP as potentially most relevant among the cerebellar peduncles in relation to stuttering: while speculative, weaker white matter development in the right ICP during childhood may lead to possible maladaptive changes in the homologous left ICP that is linked to slower speech rate that may be a compensatory process associated with years of stuttering.

The results of the current study, which shows reduced white matter integrity in the ICP that conveys error feedback via the mossy fibers, indicate that the error monitoring function of the cerebellum may be affected in PWS, which has implications for updating internal models for adaptive motor control. The afferent fibers from the olivary nucleus relay error signals from mismatches between motor commands and peripheral input (Streng et al., 2018). Other studies have also suggested the olivo-cerebellar fibers are associated with sensorimotor adaptation (Jossinger et al., 2020) and detecting motor command errors (for a review, see Shadmehr, 2017). More specifically, the ICP carries afferent fibers from the olivary nucleus, which is one of the two main afferent inputs to the cerebellum. These olivary inputs are carried through the climbing fibers, which convey bottom-up sensory feedback-related signals. The cerebellum also receives afferent input from the pons via the mossy fibers, which relay information from cortical areas. These inputs are thought to carry efference copy information, relaying an estimate of the sensory feedback predicted from the execution of the movement. When there is a mismatch between the predicted and actual sensory feedback from the movement, the cerebellum can signal this discrepancy back to the cerebrum, resulting in adaptation of the movement so that any discrepancies between 
predicted and actual sensory feedback can be minimized in future movements. In a recent review paper on altered feedback studies in stuttering, the authors note that while people who stutter seem to show less compensation to sudden perturbation, they were particularly impaired in tasks that required adaptation of motor behavior that is induced by sustained perturbation of sensory feedback (Bradshaw et al., 2021). Of the two types of responses induced by perturbation tasks - automatic compensation to sudden/unexpected perturbation vs. adaptation in response to sustained perturbation-it is the latter in which cerebellar activity is most reliably evoked (Johnson et al., 2019). Namely, the cerebellum plays a critical role in motor adaptation that enables adjusting predictions for subsequent behavior and enabling fine-tuning of behavior based on feedback. Collectively, the findings reviewed here suggest that altered performance on adaptation studies reported in people who stutter may be linked to deficits in cerebellar error monitoring and/or signaling to the cerebrum for adaptive changes in response to sensory feedback perturbation.

Developmental stuttering is often connected to aberrant error monitoring (Max et al., 2004; Postma \& Kolk, 1992). Some influential theoretical perspectives have suggested that people who stutter have aberrant internal models, which integrate prediction of the state of the speech system as a result of motor plans, and sensory feedback that results from speech (Max et al., 2004). The cerebellum is hypothesized to build associations between sensory states of speech and the motor cortex during development (for a review, see Tourville \& Guenther, 2011) and support learning associated with inverse models (e.g., Kawato, 1999). Structural differences in this pathway in children who stutter suggest that the development of internal models may be atypical. Auditory perturbation studies have found that adults who stutter are unable to update their motor commands in response to errors in their feedback (Cai et al., 2012, 2014; Daliri et al., 2017; Daliri \& Max, 2018; Kim et al., 2020; Loucks et al., 2012; Sares et al., 2018). Previous behavioral studies of auditory perturbation have variable results in children who stutter (Daliri et al., 2017; Kim et al., 2020). The current AFQ analyses suggest that cerebellar pathways associated with error monitoring are reduced in white matter integrity in children who stutter and that the higher the stuttering severity, the lower the white matter integrity in the same tracts. Structural differences in the ICP that carry olivo-cerebellar tracts may lead to variable error detection signals during speech, resulting in atypical updating of motor plans and less compensatory behaviors during perturbation trials. Additionally, associations between the frequency of stuttering and FA of the right ICP in CWS further suggest that structural differences in this pathway may contribute to greater frequency of disfluencies in speech.

Disruptions to error detection systems of the cerebellum may result in atypical speech motor control development. Reduced compensation and adaptation responses to auditory perturbation have been consistently reported in adults who stutter (Cai et al., 2012, 2014; Daliri et al., 2017; Daliri \& Max, 2018; Kim et al., 2020; Loucks et al., 2012; Sares et al., 2018), but not as consistently for children who stutter (Daliri et al., 2017; Kim et al., 2020) may at least be partially be explained by changes in the speech motor control systems over a lifetime of stuttering in people who stutter (Cai et al., 2012, 2014; Daliri et al., 2017; Daliri \& Max, 2018; Kim et al., 2020; Loucks et al., 2012; Sares et al., 2018). Over time, an inability for the cerebellum to efficiently detect errors used for error-based learning may lead to atypical internal model development that may be especially evident in adults who stutter. Alternatively, a recent study by Kim et 
al. (2020) suggests that when feedback alterations induce motor adjustments to aspects of vocal tract configuration (requiring intrinsic movement control), decreases in adaptation were pronounced in young children who stutter relative to age-matched controls. This result contrasts with those reported by Daliri et al. (2017), which showed that while significantly reduced adaptation was observed in adults who stutter compared to controls, children who stutter did not differ from controls in adaptation extent. However, the latter study used feedback perturbations that altered perception of the phoneme (vowel target), which may have led to conscious adjustments to one's speech. The difference between the more implicitly controlled speech perturbation paradigm in Kim et al. (2020) compared to Daliri et al. (2017), which may rely on explicit aspects of adaptation, could have led to the discrepancies between the two studies. Kim et al. (2020) argue that motor adaptation that involves implicit, rather than explicit, learning components might differ in people who stutter, regardless of age. Differences in implicit motor adaptation are present early in development rather than acquired later in life. Motor adaptation in response to feedback alterations that can be consciously monitored, however, may become more evident with age. Conscious motor adaptations rely on additional neural resources outside of the cerebellum, including attention networks. It may be that functional differences in these circuits differentiated adults from controls, but not between children who stutter and controls (Daliri et al., 2017). The implicit motor adaptation to selfinitiated movement that occurs automatically and is mostly outside of conscious control, however, is supported by the cerebellum (Knolle et al., 2013). Therefore, the extent of data from published studies in stuttering suggests that motor adaptation deficits are present in both children and adults who stutter when implicit learning is involved, indicating that this may be a core characteristic in developmental stuttering. Namely, atypical internal model development reflected in altered cerebellar morphology could affect implicit motor adaptation starting in childhood rather than a process acquired later over a lifetime of stuttering. Because few studies have examined children to date on perturbation experiments, it is essential to conduct more studies that use perturbation paradigms that induce both implicit and explicit adaptation mechanisms to confirm whether children close to stuttering onset indeed primarily exhibit deficits in implicit motor learning. This finding would align with the current results that show that children who stutter have reduced integrity of white matter tracts associated with error monitoring function of the cerebellum.

In addition to significant group differences in the right ICP and its negative correlation with stuttering frequency, FA values in the right ICP showed significant age-related increases in both groups. This finding suggests that structural development of this largely afferent cerebellar pathway is ongoing at least during the age range of children we observed in this study (3-11 years). Age related FA increases were also observed in the left ICP as well as the bilateral SCP (Table 2). White matter integrity increases during preschool and school-age developmental period thus seem to be on-going in the CPs that contain afferent fibers from the periphery to the cerebellum, as well as efferent fibers from the cerebellum to subcortical and cortical regions. It may be that during this period of dynamic growth, children who stutter are particularly vulnerable to aberrant growth in the ICP, which may either result in or be influenced by aberrant error-based motor learning and error monitoring in their speech. The directionality of this relationship is unclear and requires studying greater numbers of younger CWS and longitudinal studies. 
Though AFQ presents an updated analysis method for diffusion metrics derived from dMRI, these results could be further extended and confirmed through tractography analyses using tools that have shown to be superior in resolving crossing fibers (Tournier et al., 2008; Wedeen et al., 2008; Yeatman et al., 2012). Future studies could also consider examining white matter microstructure in conjunction with functional connectivity analyses of resting state fMRI data or task-based fMRI that involve a motor learning paradigm. These approaches could further establish the links between the structure and function of cerebellar tracts that are linked to error monitoring and adaptive motor learning. In addition, future research on this topic could benefit from longitudinal analyses. Neural developmental trajectories differ between children who stutter and controls and between children who stutter who eventually recover from or persist in stuttering (e.g., Chang et al., 2018; Chow \& Chang, 2017). Future studies that examine structural development of the cerebellum, as well as functional and structural examinations of cerebellocortical networks, would further provide much-needed information on whether and how specific cerebellar lobules critical to motor control and networks change over development and how that interacts with speech motor control development and stuttering severity.

In conclusion, results from this study demonstrate that white matter integrity as reflected in fractional anisotropy (FA) measures are significantly reduced in the right ICP in children who stutter relative to agematched peers. Further, FA of the right ICP was found to be negatively correlated with the frequency of stuttering. The significant differences found in the white matter microstructure of the right ICP in developmental stuttering support theories that propose that error detection differences contribute to developmental stuttering.

\section{Abbreviations}

AFQ Automated Fiber Quantification

CP Cerebellar Peduncle

CWS Children who stutter

dMRI Diffusion MRI

EVT Expressive Vocabulary Test

FA Fractional Anisotropy

fMRI Functional Magnetic Resonance Imaging

GFTA Goldman-Fristoe Test of Articulation

MD Mean Diffusivity

ICP Inferior Cerebellar Peduncle 
MCP Middle Cerebellar Peduncle

PPVT Peabody Picture Vocabulary Test

ROI Region of Interest

SCP Superior Cerebellar Peduncle

SLD Stuttering-like Disfluency

STT Streamlines tracking tractography

WASI Wechsler Abbreviated Scale of Intelligence

WPPSI Wechsler Preschool and Primary Scale of Intelligence

\section{Declarations}

Funding (information that explains whether and by whom the research was supported)

This research was supported by the National Institute on Deafness and Other Communication Disorders grant R01DC011277 (PI Chang)

Conflicts of interest/Competing interests (include appropriate disclosures)

The authors declare that there are no conflicts of interest.

Availability of data and material (data transparency)

The datasets analyzed for this research are are available on reasonable written request and through data agreement.

Code availability (software application or custom code)

Toolboxes used for AFQ analyses are freely available at GitHub (https://github.com/yeatmanlab/AFQ). Customized portion is available on request.

Authors' contributions (optional: please review the submission guidelines from the journal whether statements are mandatory)

Conceptualization: Soo-Eun Chang, Yanni Liu; Methodology: Soo-Eun Chang, Yanni Liu, Noah Waller; Formal analysis and investigation: Soo-Eun Chang, Yanni Liu, Noah Waller, Chelsea Johnson; Writing original draft preparation: Chelsea Johnson, Soo-Eun Chang, Yanni Liu; Writing - review and editing: SooEun Chang, Chelsea Johnson, Yanni Liu, Noah Waller; Funding acquisition: Soo-Eun Chang; Supervision: Soo-Eun Chang, Yanni Liu. 
Ethics approval (include appropriate approvals or waivers)

All procedures were approved by the Michigan State University Institutional Review Board

Consent to participate (include appropriate statements)

Written informed consent was obtained from one parent of the participants and assent from the participant prior to participation.

Consent for publication (include appropriate statements)

All authors approved this submission.

\section{References}

1. Akkal D, Dum RP, Strick PL (2007) Supplementary Motor Area and Presupplementary Motor Area: Targets of Basal Ganglia and Cerebellar Output. J Neurosci 27(40):10659-10673. https://doi.org/10.1523/JNEUROSCI.3134-07.2007

2. Beal DS, Gracco VL, Lafaille SJ, De Nil LF (2007) Voxel-based morphometry of auditory and speechrelated cortex in stutterers. NeuroReport 18(12):1257. https://doi.org/10.1097/WNR.0b013e3282202c4d

3. Bostan AC, Dum RP, Strick PL (2010) The basal ganglia communicate with the cerebellum. Proceedings of the National Academy of Sciences, 107(18), 8452-8456. https://doi.org/10.1073/pnas.1000496107

4. Bostan A, Dum RP, Strick PL (2013) Cerebellar networks with the cerebral cortex and basal ganglia. Trends in Cognitive Sciences 17(5):241-254. https://doi.org/10.1016/j.tics.2013.03.003

5. Bradshaw AR, Lametti DR, McGettigan C (2021) The Role of Sensory Feedback in Developmental Stuttering: A Review. Neurobiology of Language, 1-27. https://doi.org/10.1162/nol_a_00036

6. Brodal P (1978) The Corticopontine Projection in the Rhesus Monkey Origin and Principles of Organization. Brain 101(2):251-283. https://doi.org/10.1093/brain/101.2.251

7. Brown S, Ingham RJ, Ingham JC, Laird AR, Fox PT (2005) Stuttered and fluent speech production: An ALE meta-analysis of functional neuroimaging studies. Hum Brain Mapp 25(1):105-117. https://doi.org/10.1002/hbm.20140

8. Bruckert L, Shpanskaya K, McKenna ES, Borchers LR, Yablonski M, Blecher T, Ben-Shachar M, Travis KE, Feldman HM, Yeom KW (2019) Age-Dependent White Matter Characteristics of the Cerebellar Peduncles from Infancy Through Adolescence. The Cerebellum 18(3):372-387. https://doi.org/10.1007/s12311-018-1003-9

9. Budde KS, Barron DS, Fox PT (2014) Stuttering, induced fluency, and natural fluency: A hierarchical series of activation likelihood estimation meta-analyses. Brain Lang 139:99-107. https://doi.org/10.1016/j.bandl.2014.10.002 
10. Cai S, Beal DS, Ghosh SS, Guenther FH, Perkell JS (2014) Impaired timing adjustments in response to time-varying auditory perturbation during connected speech production in persons who stutter. Brain Lang 129:24-29. https://doi.org/10.1016/j.bandl.2014.01.002

11. Cai S, Beal DS, Ghosh SS, Tiede MK, Guenther FH, Perkell JS (2012) Weak Responses to Auditory Feedback Perturbation during Articulation in Persons Who Stutter: Evidence for Abnormal AuditoryMotor Transformation. PLOS ONE 7(7):e41830. https://doi.org/10.1371/journal.pone.0041830

12. Chang S-E, Chow HM, Wieland EA, McAuley JD (2016) Relation between functional connectivity and rhythm discrimination in children who do and do not stutter. Neurolmage: Clinical 12:442-450. https://doi.org/10.1016/j.nicl.2016.08.021

13. Chang S-E, Erickson KI, Ambrose NG, Hasegawa-Johnson MA, Ludlow CL (2008) Brain anatomy differences in childhood stuttering. Neuroimage 39(3):1333-1344

14. Chang S-E, Garnett EO, Etchell A, Chow HM (2018) Functional and Neuroanatomical Bases of Developmental Stuttering: Current Insights. The Neuroscientist, 1073858418803594. https://doi.org/10.1177/1073858418803594

15. Chang S-E, Zhu DC (2013) Neural network connectivity differences in children who stutter. Brain 136(12):3709-3726. https://doi.org/10.1093/brain/awt275

16. Chang S-E, Zhu DC, Choo AL, Angstadt M (2015) White matter neuroanatomical differences in young children who stutter. Brain 138(3):694-711. https://doi.org/10.1093/brain/awu400

17. Chow HM, Chang S-E (2017) White matter developmental trajectories associated with persistence and recovery of childhood stuttering: White Matter Development in Children Who Stutter. Hum Brain Mapp. https://doi.org/10.1002/hbm.23590

18. Connally EL, Ward D, Howell P, Watkins KE (2014) Disrupted white matter in language and motor tracts in developmental stuttering. Brain Lang 131:25-35.

https://doi.org/10.1016/j.bandl.2013.05.013

19. Craig-McQuaide A, Akram H, Zrinzo L, Tripoliti E (2014) A review of brain circuitries involved in stuttering. Frontiers in Human Neuroscience, 8. https://doi.org/10.3389/fnhum.2014.00884

20. Daliri A, Max L (2018) Stuttering adults' lack of pre-speech auditory modulation normalizes when speaking with delayed auditory feedback. Cortex 99:55-68.

https://doi.org/10.1016/j.cortex.2017.10.019

21. Daliri A, Wieland EA, Cai S, Guenther FH, Chang SE (2017) Auditory-motor adaptation is reduced in adults who stutter but not in children who stutter. Developmental Science, e12521.

https://doi.org/10.1111/desc.12521

22. De Nil LF, Kroll RM, Houle S (2001) Functional neuroimaging of cerebellar activation during single word reading and verb generation in stuttering and nonstuttering adults. Neurosci Lett 302(2):77-80. https://doi.org/10.1016/S0304-3940(01)01671-8

23. De Nil LF, Kroll RM, Lafaille SJ, Houle S (2003) A positron emission tomography study of short- and long-term treatment effects on functional brain activation in adults who stutter. Journal of Fluency Disorders 28(4):357-380. https://doi.org/10.1016/j.jludis.2003.07.002 
24. De Zeeuw Cl, Hoogenraad CC, Koekkoek SKE, Ruigrok TJH, Galjart N, Simpson JI (1998) Microcircuitry and function of the inferior olive. Trends Neurosci 21(9):391-400. https://doi.org/10.1016/S0166-2236(98)01310-1

25. Garnett EO, Chow HM, Nieto-Castañón A, Tourville JA, Guenther FH, Chang SE (2018) Anomalous morphology in left hemisphere motor and premotor cortex of children who stutter. Brain. https://doi.org/10.1093/brain/awy199

26. Glickstein M, May JG, Mercier BE (1985) Corticopontine projection in the macaque: The distribution of labelled cortical cells after large injections of horseradish peroxidase in the pontine nuclei. Journal of Comparative Neurology 235(3):343-359. https://doi.org/10.1002/cne.902350306

27. Hoover JE, Strick PL (1999) The Organization of Cerebellar and Basal Ganglia Outputs to Primary Motor Cortex as Revealed by Retrograde Transneuronal Transport of Herpes Simplex Virus Type 1. J Neurosci 19(4):1446-1463. https://doi.org/10.1523/JNEUROSCI.19-04-01446.1999

28. Hoshi E, Tremblay L, Féger J, Carras PL, Strick PL (2005) The cerebellum communicates with the basal ganglia. Nat Neurosci 8(11):1491-1493. https://doi.org/10.1038/nn1544

29. Ingham RJ, Grafton ST, Bothe AK, Ingham JC (2012) Brain activity in adults who stutter: Similarities across speaking tasks and correlations with stuttering frequency and speaking rate. Brain Lang 122(1):11-24. https://doi.org/10.1016/j.bandl.2012.04.002

30. Johnson JF, Belyk M, Schwartze M, Pinheiro AP, Kotz SA (2019) The role of the cerebellum in adaptation: ALE meta-analyses on sensory feedback error. Hum Brain Mapp 40(13):3966-3981. https://doi.org/10.1002/hbm.24681

31. Jossinger S, Kronfeld-Duenias V, Zislis A, Amir O, Ben-Shachar M (2021) Speech rate association with cerebellar white-matter diffusivity in adults with persistent developmental stuttering. Brain Structure and Function, 1-16

32. Jossinger S, Mawase F, Ben-Shachar M, Shmuelof L (2020) Locomotor Adaptation Is Associated with Microstructural Properties of the Inferior Cerebellar Peduncle. The Cerebellum 19(3):370-382. https://doi.org/10.1007/s12311-020-01116-8

33. Kawato M (1999) Internal models for motor control and trajectory planning. Curr Opin Neurobiol 9(6):718-727. https://doi.org/10.1016/S0959-4388(99)00028-8

34. Kell CA, Neumann K, Behrens M, von Gudenberg AW, Giraud A-L (2018) Speaking-related changes in cortical functional connectivity associated with assisted and spontaneous recovery from developmental stuttering. Journal of Fluency Disorders 55:135-144. https://doi.org/10.1016/j.jfludis.2017.02.001

35. Kelly RM, Strick PL (2000) Rabies as a transneuronal tracer of circuits in the central nervous system. J Neurosci Methods 103(1):63-71. https://doi.org/10.1016/S0165-0270(00)00296-X

36. Kelly RM, Strick PL (2003) Cerebellar Loops with Motor Cortex and Prefrontal Cortex of a Nonhuman Primate. The Journal of Neuroscience 23(23):8432-8444. https://doi.org/10.1523/JNEUROSCI.2323-08432.2003 
37. Kim KS, Daliri A, Flanagan JR, Max L (2020) Dissociated Development of Speech and Limb Sensorimotor Learning in Stuttering: Speech Auditory-motor Learning is Impaired in Both Children and Adults Who Stutter. Neuroscience. https://doi.org/10.1016/j.neuroscience.2020.10.014

38. Knolle F, Schröger E, Kotz SA (2013) Cerebellar contribution to the prediction of self-initiated sounds. Cortex 49(9):2449-2461. https://doi.org/10.1016/j.cortex.2012.12.012

39. Kronfeld-Duenias V, Amir O, Ezrati-Vinacour R, Civier O, Ben-Shachar M (2016a) The frontal aslant tract underlies speech fluency in persistent developmental stuttering. Brain Structure Function 221(1):365-381. https://doi.org/10.1007/s00429-014-0912-8

40. Kronfeld-Duenias V, Amir O, Ezrati-Vinacour R, Civier O, Ben-Shachar M (2016b) Dorsal and ventral language pathways in persistent developmental stuttering. Cortex 81:79-92. https://doi.org/10.1016/j.cortex.2016.04.001

41. Kronfeld-Duenias V, Civier O, Amir O, Ezrati-Vinacour R, Ben-Shachar M (2018) White matter pathways in persistent developmental stuttering: Lessons from tractography. Journal of Fluency Disorders 55:68-83. https://doi.org/10.1016/j.jludis.2017.09.002

42. Leichnetz GR, Smith DJ, Spencer RF (1984) Cortical projections to the paramedian tegmental and basilar pons in the monkey. Journal of Comparative Neurology 228(3):388-408. https://doi.org/10.1002/cne.902280307

43. Loucks T, Chon H, Han W (2012) Audiovocal integration in adults who stutter. International Journal of Language Communication Disorders 47(4):451-456. https://doi.org/10.1111/j.14606984.2011.00111.x

44. Lu C, Chen C, Ning N, Ding G, Guo T, Peng D, Yang Y, Li K, Lin C (2010) The neural substrates for atypical planning and execution of word production in stuttering. Exp Neurol 221(1):146-156. https://doi.org/10.1016/j.expneurol.2009.10.016

45. Lu C, Chen C, Peng D, You W, Zhang X, Ding G, Deng X, Yan Q, Howell P (2012) Neural anomaly and reorganization in speakers who stutter: A short-term intervention study. Neurology 79(7):625-632. https://doi.org/10.1212/WNL.0b013e31826356d2

46. Lu C, Ning N, Peng D, Ding G, Li K, Yang Y, Lin C (2009) The role of large-scale neural interactions for developmental stuttering. Neuroscience 161(4):1008-1026. https://doi.org/10.1016/j.neuroscience.2009.04.020

47. Lu C, Peng D, Chen C, Ning N, Ding G, Li K, Yang Y, Lin C (2010a) Altered effective connectivity and anomalous anatomy in the basal ganglia-thalamocortical circuit of stuttering speakers. Cortex 46(1):49-67. https://doi.org/10.1016/j.cortex.2009.02.017

48. Lu C, Peng D, Chen C, Ning N, Ding G, Li K, Yang Y, Lin C (2010b) Altered effective connectivity and anomalous anatomy in the basal ganglia-thalamocortical circuit of stuttering speakers. Cortex 46(1):49-67. https://doi.org/10.1016/j.cortex.2009.02.017

49. Mangold SA, Das M, J (2020) Neuroanatomy, Reticular Formation. In StatPearls. StatPearls Publishing. http://www.ncbi.nlm.nih.gov/books/NBK556102/ 
50. Max L, Guenther FH, Gracco VL, Ghosh SS, Wallace ME (2004) Unstable or Insufficiently Activated Internal Models and Feedback-Biased Motor Control as Sources of Dysfluency: A Theoretical Model of Stuttering. Contemporary issues in communication science disorders 31(Spring):105-122

51. Mayer M (1969) Frog, where are you? Dial Press, New York

52. Miall RC, Weir DJ, Wolpert DM, Stein JF (1993) Is the Cerebellum a Smith Predictor? J Mot Behav 25(3):203-216. https://doi.org/10.1080/00222895.1993.9942050

53. Middleton FA, Strick PL (1994) Anatomical Evidence for Cerebellar and Basal Ganglia Involvement in Higher Cognitive Function. Science 266(5184):458-461. JSTOR

54. Middleton FA, Strick PL (2001) Cerebellar Projections to the Prefrontal Cortex of the Primate. The Journal of Neuroscience 21(2):700-712. https://doi.org/10.1523/JNEUROSCI.21-02-00700.2001

55. Nichols TE, Holmes AP (2002) Nonparametric permutation tests for functional neuroimaging: A primer with examples. Hum Brain Mapp 15(1):1-25

56. Pierpaoli C, Basser PJ (1996) Toward a quantitative assessment of diffusion anisotropy. Magn Reson Med 36(6):893-906. https://doi.org/10.1002/mrm.1910360612

57. Postma A, Kolk H (1992) Error Monitoring in People Who Stutter: Evidence Against Auditory Feedback Defect Theories. Journal of Speech Language Hearing Research 35(5):1024-1032

58. Purves D, Augustine GJ, Fitzpatrick D, Hall WC, LaMantia AS, McNamara JO, Williams S (2004) Modulation of movement by the cerebellum. Neuroscience 3:435-452

59. Rhodes BJ, Bullock D (2002) A Scalable Model of Cerebellar Adaptive Timing and Sequencing: The Recurrent Slide and Latch (RSL) Model. Applied Intelligence 17(1):35-48

60. Riley GD (2009) Stuttering Severity Instrument: SSI-4. Pro-Ed, Inc

61. Rohde GK, Barnett AS, Basser PJ, Marenco S, Pierpaoli C (2004) Comprehensive approach for correction of motion and distortion in diffusion-weighted MRI. Magn Reson Med 51(1):103-114. https://doi.org/10.1002/mrm.10677

62. Sares AG, Deroche MLD, Shiller DM, Gracco VL (2018) Timing variability of sensorimotor integration during vocalization in individuals who stutter. Sci Rep 8(1):16340. https://doi.org/10.1038/s41598018-34517-1

63. Schmahmann JD, Pandya DN (1991) Projections to the basis pontis from the superior temporal sulcus and superior temporal region in the rhesus monkey. Journal of Comparative Neurology 308(2):224-248. https://doi.org/10.1002/cne.903080209

64. Schmahmann JD, Pandya DN (1993) Prelunate, occipitotemporal, and parahippocampal projections to the basis pontis in rhesus monkey. Journal of Comparative Neurology 337(1):94-112. https://doi.org/10.1002/cne.903370107

65. Schmahmann JD, Pandya DN (1997) Anatomic Organization of the Basilar Pontine Projections from Prefrontal Cortices in Rhesus Monkey. The Journal of Neuroscience 17(1):438-458. https://doi.org/10.1523/JNEUROSCI.17-01-00438.1997 
66. Shadmehr R (2017) Learning to Predict and Control the Physics of Our Movements. The Journal of Neuroscience 37(7):1663-1671. https://doi.org/10.1523/JNEUROSCI.1675-16.2016

67. Sitek KR, Cai S, Beal DS, Perkell JS, Guenther FH, Ghosh SS (2016) Decreased CerebellarOrbitofrontal Connectivity Correlates with Stuttering Severity: Whole-Brain Functional and Structural Connectivity Associations with Persistent Developmental Stuttering. Frontiers in Human Neuroscience, 10. https://doi.org/10.3389/fnhum.2016.00190

68. Smith A, Weber C (2017) How stuttering develops: The multifactorial dynamic pathways theory. Journal of Speech Language Hearing Research 60:2483-2505. https://doi.org/10.1044/2017_JSLHR-S-16-0343

69. Smith SM, Jenkinson M, Johansen-Berg H, Rueckert D, Nichols TE, Mackay CE, Watkins KE, Ciccarelli O, Cader MZ, Matthews PM, Behrens TEJ (2006) Tract-based spatial statistics: Voxelwise analysis of multi-subject diffusion data. Neurolmage 31(4):1487-1505. https://doi.org/10.1016/j.neuroimage.2006.02.024

70. Snider RS, Maiti A (1976) Cerebellar contributions to the papez circuit. J Neurosci Res 2(2):133-146. https://doi.org/10.1002/jnr.490020204

71. Song LP, Peng DL, Jin Z, Yao L, Ning N, Guo XJ, Zhang T (2007) Gray matter abnormalities in developmental stuttering determined with voxel-based morphometry. Zhonghua Yi Xue Za Zhi 87(41):2884-2888

72. Stoodley CJ, Schmahmann JD (2010) Evidence for topographic organization in the cerebellum of motor control versus cognitive and affective processing. Cortex; a Journal Devoted to the Study of the Nervous System Behavior 46(7):831-844. https://doi.org/10.1016/j.cortex.2009.11.008

73. Streng ML, Popa LS, Ebner TJ (2018) Complex Spike Wars: A New Hope. The Cerebellum 17(6):735746. https://doi.org/10.1007/s12311-018-0960-3

74. Strick PL, Dum RP, Fiez JA (2009) Cerebellum and Nonmotor Function. Annu Rev Neurosci 32(1):413-434. https://doi.org/10.1146/annurev.neuro.31.060407.125606

75. Tournier J-D, Yeh C-H, Calamante F, Cho K-H, Connelly A, Lin C-P (2008) Resolving crossing fibres using constrained spherical deconvolution: Validation using diffusion-weighted imaging phantom data. Neurolmage 42(2):617-625. https://doi.org/10.1016/j.neuroimage.2008.05.002

76. Tourville JA, Guenther FH (2011) The DIVA model: A neural theory of speech acquisition and production. Language Cognitive Processes 26(7):952-981.

https://doi.org/10.1080/01690960903498424

77. Tsang JM, Dougherty RF, Wandell BA (2010) Tract alignment errors decrease detection power in group analyses of diffusion data with TBSS. Society for Neuroscience. San Diego, CA

78. Vilensky JA, Van Hoesen GW (1981) Corticopontine projections from the cingulate cortex in the rhesus monkey. Brain Res 205(2):391-395. https://doi.org/10.1016/0006-8993(81)90348-6

79. Watkins KE, Smith SM, Davis S, Howell P (2007) Structural and functional abnormalities of the motor system in developmental stuttering. Brain 131(1):50-59. https://doi.org/10.1093/brain/awm241 
80. Wedeen VJ, Wang RP, Schmahmann JD, Benner T, Tseng WYI, Dai G, Pandya DN, Hagmann P, D'Arceuil $\mathrm{H}$, de Crespigny AJ (2008) Diffusion spectrum magnetic resonance imaging (DSI) tractography of crossing fibers. Neurolmage 41(4):1267-1277. https://doi.org/10.1016/j.neuroimage.2008.03.036

81. Wolpert DM, Miall RC, Kawato M (1998) Internal models in the cerebellum. Trends in Cognitive Sciences 2(9):338-347

82. Yairi E, Ambrose NG (1999) Early childhood stuttering I: Persistency and recovery rates. Journal of Speech Language Hearing Research 42:1097-1112

83. Yang Y, Jia F, Siok WT, Tan LH (2016) Altered functional connectivity in persistent developmental stuttering. Scientific Reports, 6. https://doi.org/10.1038/srep19128

84. Yeatman JD, Dougherty RF, Myall NJ, Wandell BA, Feldman HM (2012) Tract Profiles of White Matter Properties: Automating Fiber-Tract Quantification. PLOS ONE 7(11):e49790. https://doi.org/10.1371/journal.pone.0049790

85. Zemanick MC, Strick PL, Dix RD (1991) Direction of transneuronal transport of herpes simplex virus 1 in the primate motor system is strain-dependent. Proceedings of the National Academy of Sciences, 88(18), 8048-8051. https://doi.org/10.1073/pnas.88.18.8048

\section{Figures}

a

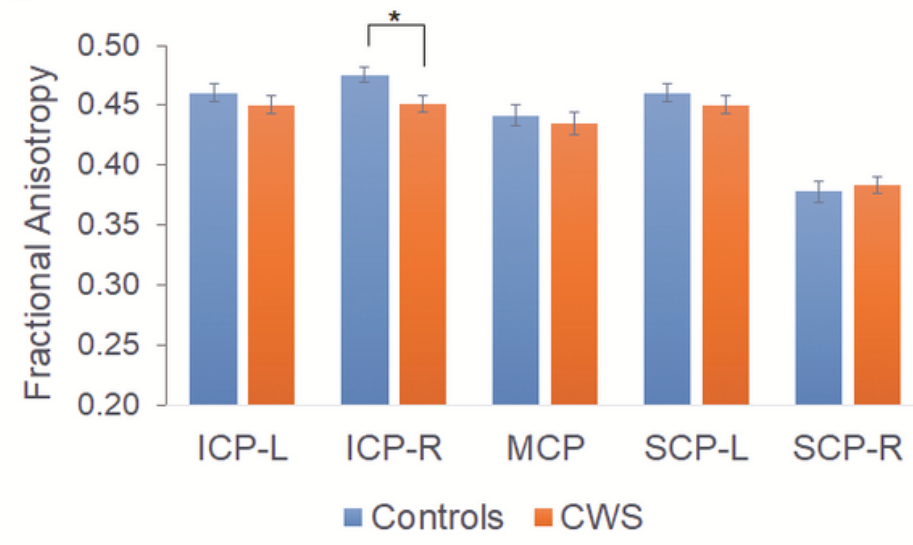

b

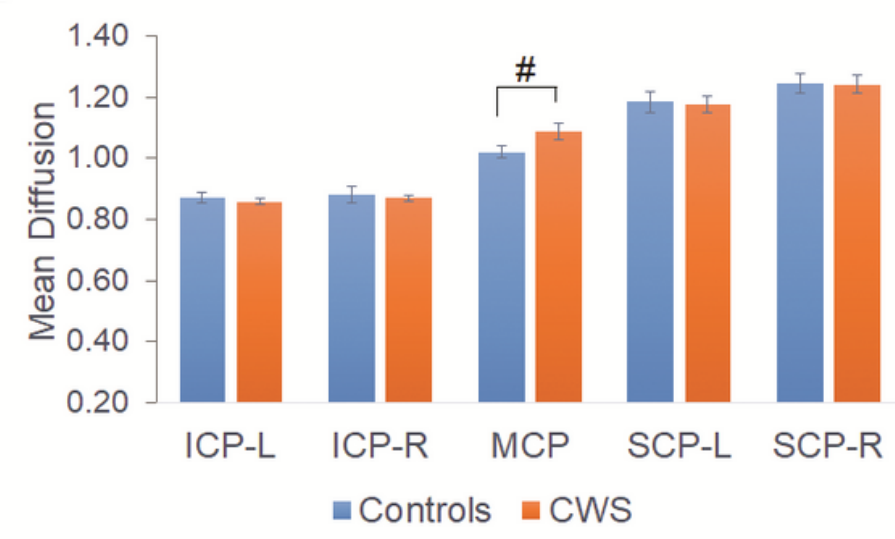

\section{Figure 1}

Mean tract_FA (a) and tract_MD (b) for all cerebellar peduncle tracts examined in children who stutter (CWS) and controls. Group difference was only significant in the FA values of the RICP. ICP = Inferior Cerebellar Peduncle, $\mathrm{MCP}=$ Middle Cerebellar Peduncle, $\mathrm{SCP}=$ Superior Cerebellar Peduncle; $\mathrm{L}=$ left; $\mathrm{R}=$ right. * $p<0.05 ; \# p<0.10$. Standard error bar is denoted. 
a

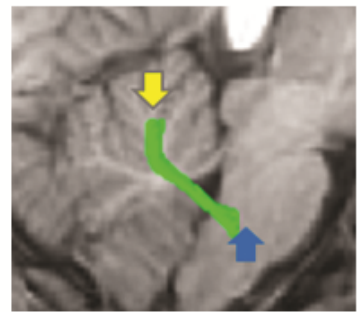

b

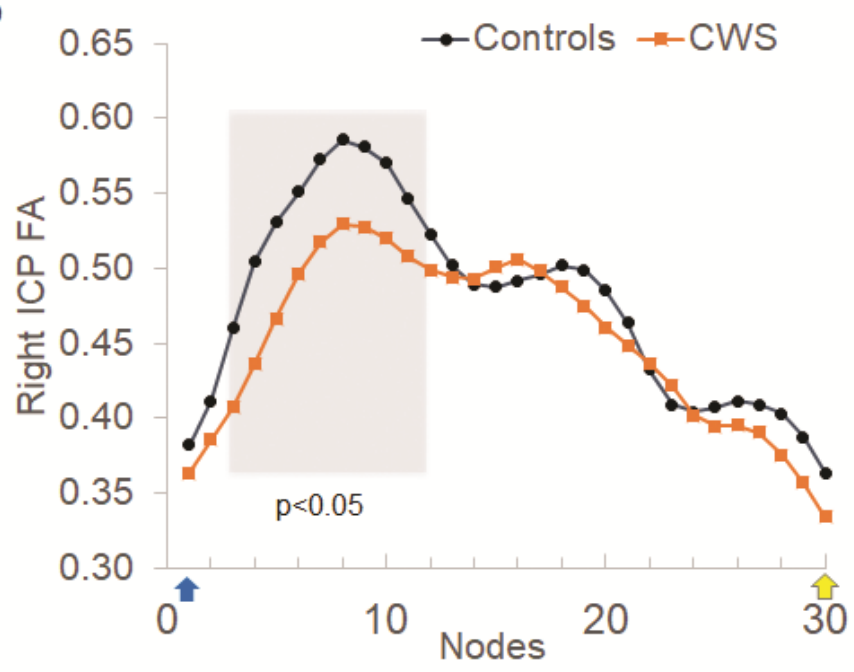

C

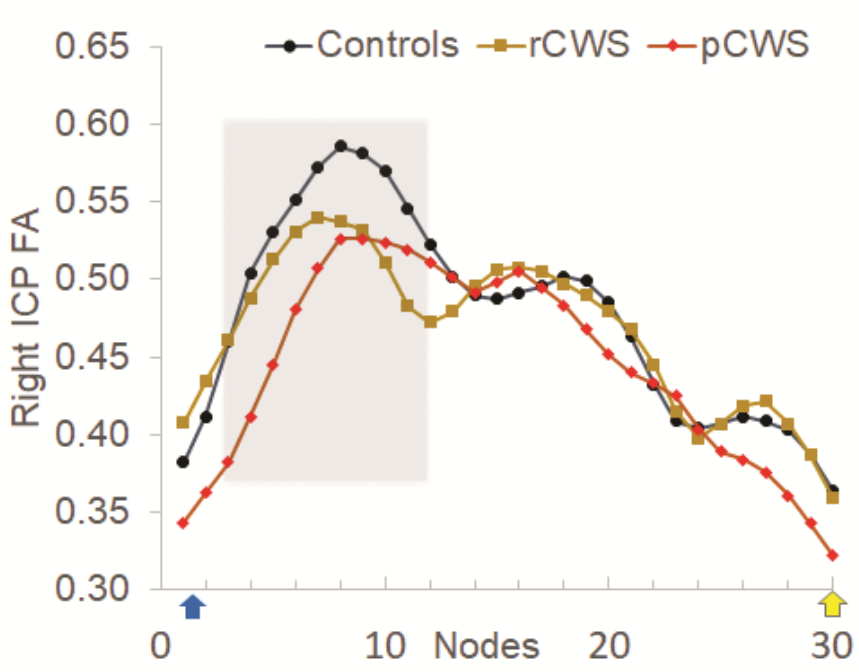

Figure 2

Group differences in FA shown along the tract nodes of the right ICP (a). Local FA of nodes 3 to 12 showed significant reduction in children who stutter (CWS) relative to controls (b). The reduction was driven by persistent CWS (c). ICP, inferior cerebellar peduncles. rCWS, recovered CWS; pCWS, persistent CWS.

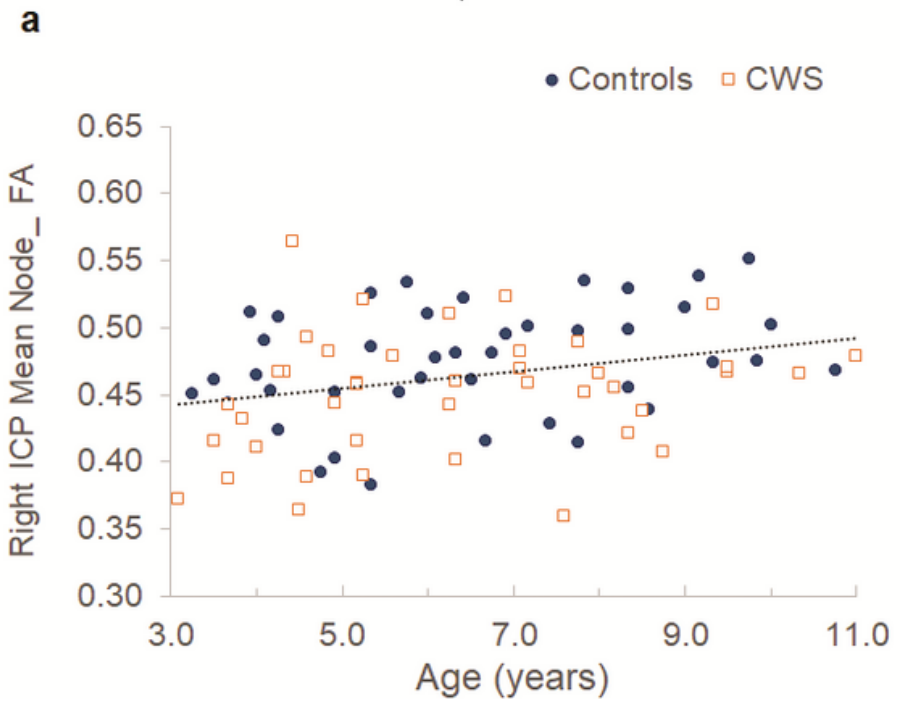

b

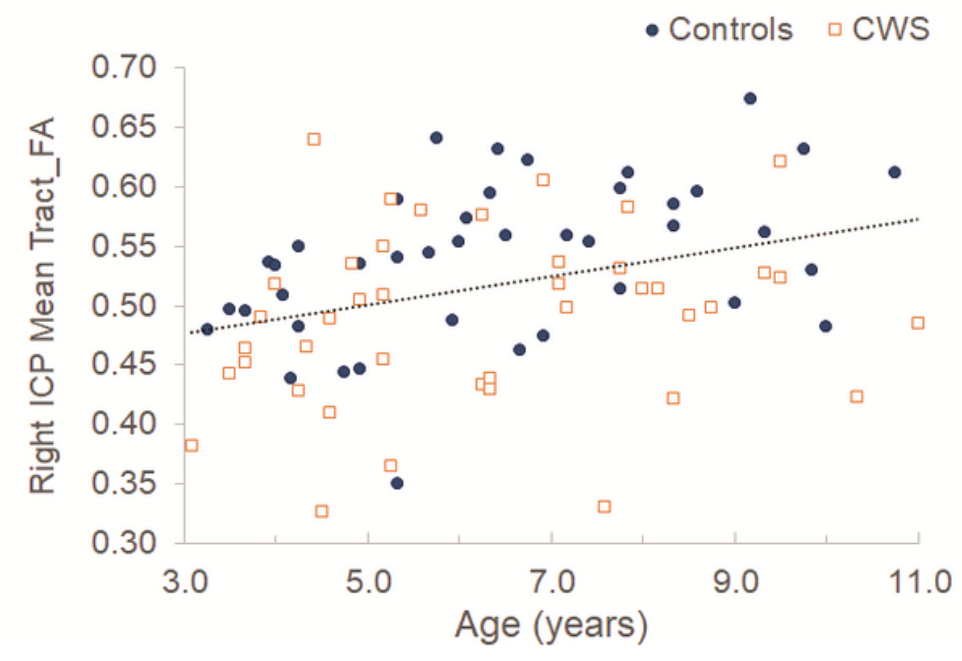

Figure 3 
Both right ICP (a) Node_FA (mean FA for nodes 3-12) and (b) Tract_FA (mean FA for the whole tract) showed a significant correlation with age regardless of group. CWS was denoted by empty square and controls was denoted by filled circle. FA: Fractional Anisotropy; ICP, inferior cerebellar peduncle.

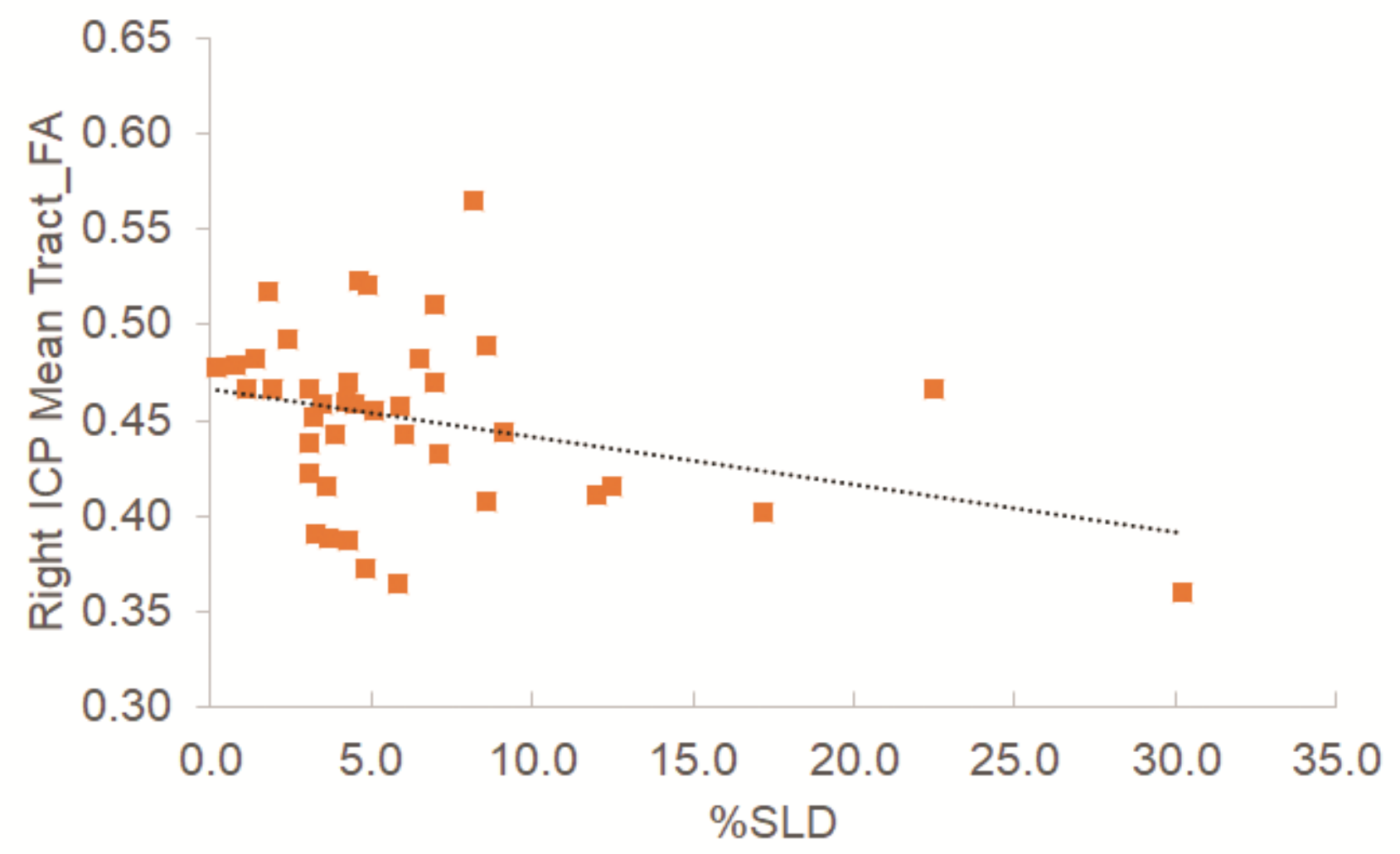

\section{Figure 4}

Stuttering frequency (\%SLD) is correlated with the tract_FA of the right ICP. Greater stuttering frequency was associated with lower tract_FA in the right ICP for children who stutter.

\section{Supplementary Files}

This is a list of supplementary files associated with this preprint. Click to download.

- Fig1s.tif 